\title{
Cómo generan y gestionan contenidos los estudiantes de educación de Costa Rica: una contribución al estudio de su entorno personal de aprendizaje
}

\author{
José Antonio García Martínez \\ jose.garcia.martinez@una.cr \\ Universidad Nacional de Costa Rica, Costa Rica \\ Mercedes González Sanmamed \\ mercedes@udc.es \\ Universidad de A Coruña, España
}

\begin{abstract}
Resumen
Descentralizar el aprendizaje de la institución formadora para que el estudiantado sea protagonista de su propio proceso es una acepción pedagógica vinculada a los Entornos Personales de Aprendizaje (PLE). El objetivo de este estudio es analizar tanto las herramientas que utilizan para crear contenido los estudiantes de último año de carreras de Educación de la Universidad Nacional de Costa Rica, como las finalidades y actividades que se generan con su uso.

En el marco de un diseño ex post facto y transeccional, se aplicó un cuestionario construido ad hoc a una muestra de 381 estudiantes. Como principales hallazgos cabe destacar el escaso conocimiento $y$, por ende, la baja utilización, que los estudiantes realizan de ciertas herramientas de la web 2.0 para crear contenido. Se constata una mayor preferencia por los recursos de escritorio más tradicionales -como procesadores de texto y presentaciones, lo que refleja un PLE limitado y poco evolucionado en el que no se aprovechan suficientemente las potencialidades que ofrece la tecnología en el momento actual.
\end{abstract}

\section{Palabras clave}

Entorno personal de aprendizaje; aplicaciones Web 2.0, tecnología educativa, estudiantes universitarios. 


\title{
How do Costa Rican education students generate and manage content: a contribution to the study of their personal learning environment
}

\author{
José Antonio García Martínez \\ jose.garcia.martinez@una.cr \\ Universidad Nacional de Costa Rica, Costa Rica \\ Mercedes González Sanmamed \\ mercedes@udc.es \\ Universidad de A Coruña, Spain
}

\begin{abstract}
Decentralizing the learning of the training institution so that the students are the protagonist of their own process, is a pedagogical meaning linked to the Personal Learning Environments (PLE). The objective of this study is to analyze both the tools to create content used by senior students in education careers from the National University of Costa Rica as the aims and activities generated by their use.

In the framework of an ex post facto and transectional design, an ad hoc constructed questionnaire was applied to a sample of 381 students. As main findings, we can foreground the lack of knowledge and, therefore, the low use, that students make of certain web 2.0 tools to create content. There is a verified greater preference for more traditional desktop resources - such as word processors and presentations - what reflects a limited and little evolved PLE, in which isn't being used sufficiently the potential offered by technology in today's.
\end{abstract}

\section{Keywords}

Personal learning environment; Web 2.0; information and communication technologies; university students. 


\section{Introducción}

Actualmente, existen un gran número de herramientas que permiten que cualquier persona pueda acceder a la información, procesarla e incluso crearla, así como difundirla de forma rápida y sencilla. Específicamente, en el ámbito educativo, se valora muy positivamente la utilidad de las tecnologías de la información y la comunicación (TIC) y, concretamente, se reconocen las potencialidades de las herramientas web 2.0 de manera que los agentes intervinientes en los distintos ámbitos educativos las están adoptando para el proceso de formación y los propios sujetos en formación las van incorporando en sus mecanismos de aprendizaje.

Algunas de estas herramientas permiten la creación de blogs, wikis, posters digitales, líneas del tiempo, hojas de texto o de cálculo, etc., donde el estudiante no solamente puede buscar información, sino crear, gestionar y compartir conocimiento con otros usuarios (Abdelrahman, AbdelAlmuniem \& Almabhouh, 2016). Esta riqueza de recursos generan un entorno propicio para que el colectivo discente sea capaz de seleccionar aquellas herramientas que se adapten en mayor medida a su estilo de aprendizaje (Atwell, 2007), lo que sin duda está provocando cambios sustanciales en la alfabetización del estudiantado una vez que se abre la posibilidad de que también ellos se conviertan en potenciales constructores de contenidos (Erstad, 2015).

El abanico de opciones del que dispone cada persona para orientar y regular su aprendizaje es ahora mucho más amplio y diverso. Y este aspecto reclama, entre otros, una revisión de los sistemas y procesos de formación tradicionales, y sugiere descentralizar las estrategias didácticas de la institución para atender a las propias necesidades de los estudiantes. Así, investigaciones al respecto (Van Den Beemt y Diepstraten, 2016; Voogt, 2010), indican que las personas que hacen un uso educativo intensivo de las TIC, no solo se sienten más seguras sino que muestran mejores actitudes hacia el aprendizaje a lo largo de la vida.

Este protagonismo del estudiantado, puede nutrirse de las ventajas que aportan las herramientas tecnológicas, especialmente en la formación de formadores, que podrán convertirse en agentes de cambio, motivadores y facilitadores, para que futuros estudiantes alcancen las habilidades y competencias tecnológicas (Roig, Mengual-Andrés, Sterrantino y Quinto, 2015) necesarias para la autonomía y autocontrol de su aprendizaje, así como potencializar el conocimiento y uso de las TIC en los procesos de enseñanza de manera eficiente (Cabero, Roig-Vila y Mengual-Andrés, 2017).

Es evidente que la incorporación de la tecnología al sistema educativo, particularmente a la educación superior, está generando cambios y replanteamientos sobre la forma de enseñar y aprender (Dabbagh y Reo, 2011), así como creando una senda de espacios no formales e informales de formación, donde el estudiantado satisface a través de internet muchas de sus necesidades de aprendizaje (Şahin \& Uluyol, 2016; Arul-Sekar y Arul-Lawrence, 2015), tanto en lo referente a la búsqueda de información, reflexión y creación de contenidos, como en la comunicación con los demás usuarios. Estos aspectos integran los denominados Personal Learning Enviroment (PLE) que, según Adell y Castañeda (2010), se definen como "(...) el conjunto de herramientas, fuentes de información, conexiones y actividades que cada persona utiliza de forma asidua para aprender" (p.23).

Si bien podría decirse que los entornos de aprendizaje existen desde siempre, cabe recordar que hasta hace unas décadas la formación estaba focalizada en las aulas y será a partir de la aparición 
de internet y la evolución a la web 2.0 cuando la información quede descentralizada y disgregada en múltiples formatos, donde cualquier persona con acceso a la red puede tanto obtener información como generarla (Castañada y Adell, 2013), gracias a los diferentes recursos disponibles. Así, el PLE constituye "un espacio social horizontal y rico en fuentes de información (red social donde el conocimiento no está cerrado) que supone una alternativa a la jerarquización y unidirecionalidad tradicional de los entornos formativos" (Fernández y Cejudo, 2009, p.111).

Pero no solo las herramientas forman el PLE, sino también las finalidades y actividades que se generan con ellas (Adell y Castañeda, 2010), tanto para buscar información, como para crear contenido y gestionarlo, así como para compartirlo con los demás. Es decir, se puede incorporar al PLE una herramienta específica, pero además se debe tener en cuenta para qué se utiliza -por ejemplo, sintetizar ideas- y que experiencias se generan con su uso -elaboración de mapas conceptuales-.

Desde su inicio, el PLE ha sido motivo de múltiples investigaciones que versan sobre el concepto y estructura del mismo (Fiedler \& Väljataga, 2013; Fiedler \& Väljataga, 2010; Väljataga y Laampere, 2010; Adell y Castañeda, 2010; Atwell, 2007) y que dejan entrever que no se trata solamente de un conjunto de herramientas específicas, sino de un nuevo paradigma donde el aprendizaje colaborativo y la creación de redes es vital para que el usuario lidere su aprendizaje a lo largo de la vida.

Actualmente los estudios sobre PLE se pueden dividir en dos grandes grupos. El primero enmarca los estudios que centran su atención en las herramientas (Sahin y Uluyol, 2016; Rahimi, Berg \& Veen, 2012, 2015, Saadatmand \& Kumpulainen, 2012; Johnson y Liber, 2008), así como en las experiencias de proyectos dirigidos a la implementación de los mismos (Gallego-Arrufat y ChavesBarboza, 2012; Marín, Linaza y Salinas, 2014; Harris, Earl, Beale, Phethea, \& Brughmans, 2012, Kop \& Fournier, 2013)

En el segundo grupo se encuentran aquellos estudios que dirigen su atención en la pedagogía de los PLE, analizando aspectos concretos como los cambios que generan hacia el aprendizaje abierto, flexible y a lo largo de la vida (Colmenero, Pérez, \& Gutiérrez, 2015; Torres y Costa, 2013; Buendía, Olmedo y González, 2009; Barboza, Torres y Nuñez, 2016), o los cambios de rol tanto del colectivo docente como discente (Sahin y Uluyol, 2016).

Generar y gestionar contenidos es una actividad de suma importancia para el estudiantado universitario que, en el marco de los estudios sobre los PLE, puede verse favorecida desde dos vertientes: por un lado, tomando en cuenta la envergadura y desarrollo de los PLE, en los cuales el conocimiento y uso de los distintos recursos ofrecerá mayores posibilidades para la elaboración de contenidos, potencializando la expresión creativa para modificar o crear nuevos contenidos (Vazquez-Cano, Martín-Monje \& Castrillo de Larreta-Azelain, 2016); y, por otro lado, gracias a la incorporación de las TIC, especialmente de la web 2.0, que haga la institución formadora (Boza y Conde, 2015), por ejemplo, a través del Learning Management Systems (LMS).

Algunas investigaciones (Atwell, 2007; Cabero y Marín, 2011; Ruiz, Sánchez y Gómez-García, 2013; Venkatesh, Croteau y Rabah, 2014, Boza y Conde, 2015) aluden a ciertas herramientas habituales dentro de los PLE que ayudan a la creación y gestión de la información obtenida previamente, entre las que destacan los blogs, wikis y redes sociales, y que son utilizadas tanto en 
trabajo individual como colaborativo. Investigaciones como la de Sahin y Uluyol (2016) enfatizan que las principales herramientas que usa el estudiantado son el correo electrónico, las redes sociales, el uso compartido de documentos, el intercambio de vídeos, los motores de búsqueda y las enciclopedias. Dichos recursos son utilizados mayoritariamente para la búsqueda e intercambio de información, destacando el bajo nivel de recursos para la construcción de contenido (Vicent, Calatayud, Perera y Rodríguez, 2017).

Independientemente de las herramientas que utiliza el colectivo discente, lo que es evidente es que la gran mayoría acceden a la universidad con cierta alfabetización digital (Gisbert y Esteve, 2016) lo que implica el conocimiento y utilización de algunos recursos tecnológicos, que no necesariamente queda ligado al desarrollo de los PLE, ya que no necesariamente suelen asociarse a contextos de aprendizaje (García, Fallas y Gamboa, 2016; Escofet, López y Álvarez, 2014)

\section{Objetivos del estudio}

El presente trabajo ${ }^{1}$ pretende analizar los PLE del estudiantado universitario del último año de carrera del Centro de Investigación en Docencia y Educación (CIDE) de la Universidad Nacional de Costa Rica, identificando, específicamente, aquellas herramientas, finalidades y actividades que el colectivo discente utiliza y genera, dentro del componente de creación y gestión de contenidos.

Para ello se plantean los siguientes objetivos específicos:

- Identificar las principales herramientas tecnológicas que el colectivo discente incorpora en sus PLE para la creación y gestión de contenidos, así como las finalidades y actividades que se generan.

- Conocer la frecuencia de uso con la que el estudiantado utiliza las herramientas para crear y gestionar contenidos.

- Identificar la percepción sobre el nivel de capacitación que tiene el estudiantado en el uso de estas herramientas tecnológicas para crear y gestionar contenidos.

Además del cumplimiento de los objetivos expuestos, se pretende dar respuesta a las preguntas que aparecen en la tabla 1, las cuáles han sido agrupadas de acuerdo a diferentes variables, tanto sociodemográficas como referentes al acceso que tiene el estudiantado a recursos tecnológicos, y la formación previa acerca de la TIC.

\footnotetext{
${ }^{1}$ El trabajo que se presenta pertenece a una investigación más extensa, cuyo objetivo fue el análisis de los PLE del estudiantado universitario del CIDE.
} 
Cómo generan y gestionan contenidos los estudiantes de educación de Costa Rica: una contribución al estudio de su entorno personal de aprendizaje

\begin{tabular}{|c|c|}
\hline VARIABLE INDEPENDIENTE & $\begin{array}{c}\text { VARIABLE DEPENDIENTE: } \\
\text { Subescala "Creación y gestión de contenidos" }\end{array}$ \\
\hline Sexo & $\begin{array}{l}\text { ¿Existen diferencias en la subescala del estudiantado } \\
\text { según el sexo? }\end{array}$ \\
\hline Edad & $\begin{array}{l}\text { ¿Existen diferencias en la subescala del estudiantado } \\
\text { según la edad? }\end{array}$ \\
\hline $\begin{array}{l}\text { Recursos tecnológicos: } \\
\text { Posesión de computadora propia } \\
\text { Acceso a internet en lugar de } \\
\text { residencia }\end{array}$ & $\begin{array}{l}\text { ¿Está relacionado el acceso a recursos tecnológicos por } \\
\text { parte del estudiantado con lo obtenido en la subescala? }\end{array}$ \\
\hline $\begin{array}{l}\text { Formación TIC: } \\
\text { Curso TIC independiente } \\
\text { Curso TIC secundaria } \\
\text { Curso TIC universidad } \\
\text { Autoformación }\end{array}$ & $\begin{array}{l}\text { ¿Existe relación entre la formación previa en TIC y la } \\
\text { puntuación obtenida en la subescala? }\end{array}$ \\
\hline
\end{tabular}

Tabla 1. Variables dependientes e independientes, y preguntas clave en torno a estas.

Fuente: Elaboración propia

\section{Método}

Para responder a las preguntas de investigación propuestas, el enfoque metodológico del estudio ha sido cuantitativo, con un alcance exploratorio-descriptivo y un diseño ex post facto, donde la recolección de datos se realiza en un solo momento, por lo que posee un carácter transversal dentro de los estudios de desarrollo (Bisquerra, 2014, Hernández, Fernández y Baptista, 2010).

\section{a. Elaboración y aplicación del cuestionario}

Teniendo en cuenta los objetivos propuestos, se eligió un estudio por encuesta (Bisquerra, 2014), concretamente a través de un cuestionario ad hoc denominado "PLEstudiantes", que se ha elaborado a partir de los componentes del PLE que proponen Castañeda y Adell $(2011,2013)$.

En este trabajo se expondrá exclusivamente el apartado referido a las herramientas (19 ítems), finalidades (11 ítems) y actividades (10 ítems) para generar y gestionar contenidos, correspondiente al bloque 4. Para la recogida de datos acerca de las herramientas utilizadas se utilizó una escala tipo Likert de cinco puntos a través de la que se recoge información sobre el conocimiento y frecuencia de uso (1 nunca hasta 5 siempre; 0 no la conozco) y nivel de capacitación en torno a las mismas ( 1 muy poco capacitado hasta 5 muy capacitado). En cuanto a las finalidades y actividades que se realizan en torno a los PLE señalar que se han valorado también con una escala de 1 (nunca) hasta 5 (siempre). Cabe destacar que se ofrece la posibilidad a la persona encuestada de reflejar otras opciones en todos los apartados a través de preguntas abiertas.

La estructura del instrumento es el resultado de un proceso de validación de contenido llevada a cabo a través de juicio de expertos (Cohen y Manion, 1990; Mc Millan y Schumacher, 2005): concretamente seis profesionales tanto del área de metodología de investigación como de tecnología educativa, los cuales valoraron las propuestas de ítems planteadas por los investigadores para medir las variables. También se realiza una prueba piloto con estudiantes de características similares a la población de estudio de la cual se obtienen aportes para mejorar la estructura y el contenido que son incorporados en el instrumento definitivo. 
Los ítems validados fueron incluidos en el cuestionario y se sometieron a las pruebas de Correlación de Pearson entre cada componente y la escala total que, como se observa en la tabla 2 , es significativa en todos los casos.

\begin{tabular}{|l|c|c|c|c|}
\hline & Escala Reflexión & Herramientas & Actividades & Finalidades \\
\hline Escala Reflexión & 1 &, $905^{* *}$ &, $734^{* *}$ &, $624^{* *}$ \\
\hline Herramientas &, $905^{* *}$ & 1 &, $565^{* *}$ &, $382^{* *}$ \\
\hline Actividades &, $734^{* *}$ &, $565^{* *}$ & 1 &, $540^{* *}$ \\
\hline Finalidades &, $624^{* *}$ &, $382^{* *}$ &, $540^{* *}$ & 1 \\
\hline
\end{tabular}

**. La correlación es significativa al nivel 0,01 (bilateral).

Tabla 2. Matriz de correlaciones ítem escala e ítem componentes Fuente: Elaboración propia

En referencia a la fiabilidad, se ha realizado el cálculo de consistencia interna Alpha de Cronbach para la escala correspondiente a "Herramientas, finalidades y actividades para generar y gestionar el contenido" obteniendo un $\alpha=0,918$, considerado por la teoría como adecuado (Cronbach, 1951), por lo que la escala utilizada puede calificarse como fiable.

\section{b. Identificación de la muestra}

La población de estudio la conforma el colectivo de estudiantes de último año de carrera del CIDE de Bachillerato y Licenciatura, así como del último trimestre de Maestría, del Campus Omar Dengo de la Universidad Nacional de Costa Rica.

Para el tamaño de la muestra se ha utilizado la fórmula de cálculo para poblaciones finitas (Arnal, del Rincón y Latorre, 1992):

$$
\mathrm{n}=\frac{\mathrm{N} \mathrm{Z} \mathrm{Z}_{\alpha}^{2} \mathrm{pq}}{\mathrm{d}^{2 *}(\mathrm{~N}-1)+\mathrm{Z}_{\alpha}^{2} \mathrm{pq}}
$$

$$
\begin{aligned}
& N=\text { total de la población } \\
& d=\text { error máximo aceptable (en este caso } 3 \%=0.03 \text { ) } \\
& z=1,96 \text { (nivel de confianza } 95 \%) \\
& p q=\text { proporción esperada; } p=(5 \%): 0,05 ; q=1-p(1-0.05=0.95)
\end{aligned}
$$

Al sustituir los datos en la fórmula se obtiene el siguiente tamaño de muestra:

$$
\mathrm{n}=\frac{2333 *(1.96)^{2} * 0.05 * 0.95}{(0.03)^{2} *(2333-1)+(1.96)^{2} * 0.05 * 0.95}=186.61=187
$$

Se obtiene un resultado de 187 estudiantes como mínimo para que la muestra sea representativa. Cabe resaltar que se obtuvieron 395 cuestionarios que, después de la revisión visual y la introducción de datos a la matriz, se redujeron a 381, número muy superior al requerido según la fórmula de cálculo aplicado, lo que indica que el tamaño de muestreo es adecuado. 
La elección de la muestra ha sido de tipo probabilístico entendida como aquella donde todos los elementos que componen la población, tienen la misma posibilidad de ser elegidos (Hernández et al., 2010), y determinada a partir de aspectos como el problema, objetivos y el diseño de la investigación propuesta. La recogida de datos se realiza a través de un cuestionario autoadministrado, pero teniendo en cuenta la selección muestral estratificada (Hernández et al., 2010; Bisquerra, 2014) donde los estratos están conformados por las Unidades Académicas que componen el CIDE y, dentro de estas, las carreras y los niveles de Bachillerato, Licenciatura y Maestría.

De cara a posibilitar la representatividad -que permitirá generalizar los datos obtenidos al resto de la población-, se ha alcanzado un porcentaje muestral muy similar al de la población (figura 1)

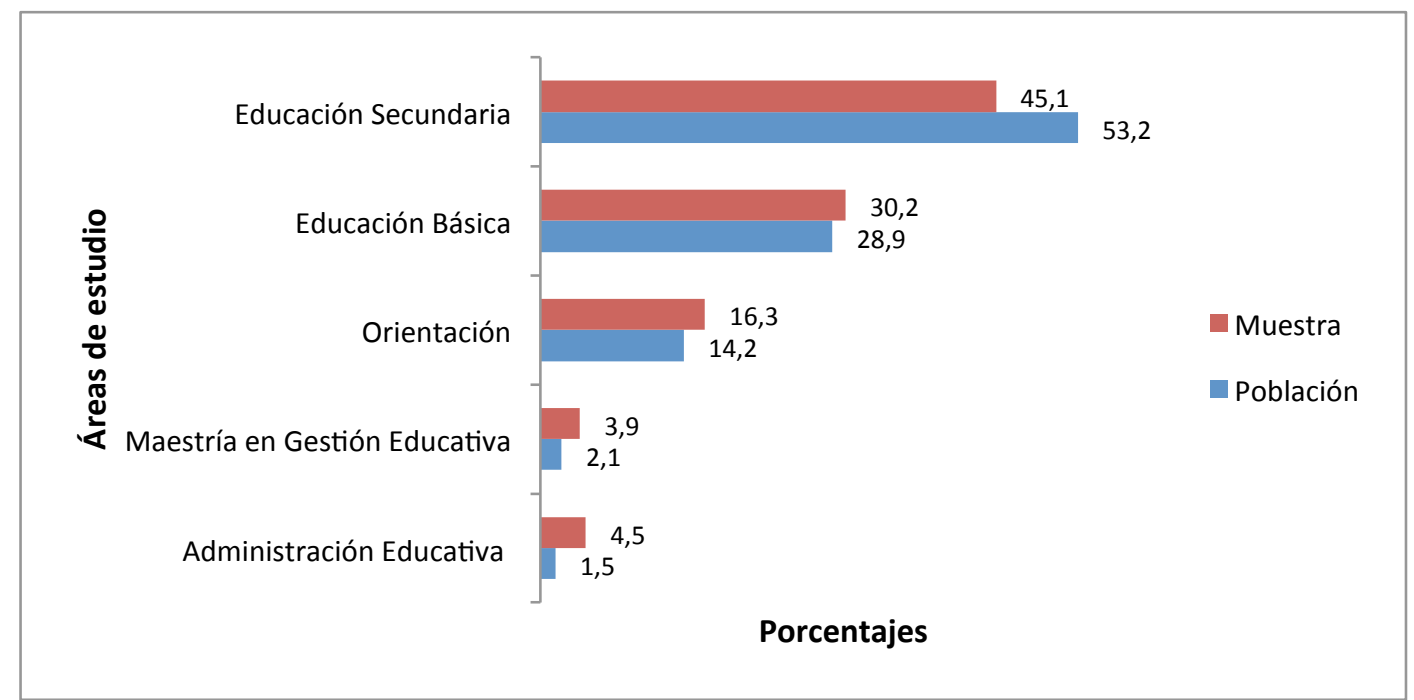

Figura 1. Comparación entre población y muestra por áreas de estudios.

Fuente: Elaboración propia con base a datos ofrecidos por la Administración del CIDE.

La edad de la muestra (Figura 2) se encuentra en un rango entre los 19 y los 58 años ( $\mathrm{Md}=21$; Media=24; DS=5,9). En cuanto al sexo del grupo participante un $84,8 \%$ son mujeres (323), mientras que el $15,2 \%$ son hombres (58). En cuanto a la distribución por grado, $243(63,8 \%)$ se ubican en Bachillerato, 123 (32,3\%) cursan Licenciatura y, los restantes 15 (3.9\%) pertenecen a una Maestría. 




Figura 2. Sexo del colectivo de estudiantes según rango de edad.

Fuente: Elaboración propia

\section{Resultados}

\section{a. Principales herramientas tecnológicas que el estudiantado incorpora en sus PLE para generar y gestionar el contenido}

Una vez realizada la búsqueda de información, el colectivo discente utiliza diferentes herramientas para crear nuevo contenido y gestionarlo. Como se observa en la figura 3, las herramientas menos conocidas y por ende menos utilizadas, son las pertenecientes a la web 2.0, donde recursos como newsletters son conocidos por un $13,4 \%$, y otras herramientas para realizar líneas temporales y póster digitales tan solo las conoce una cuarta parte del grupo encuestado. En cuanto a recursos que se usan para la elaboración de mapas conceptuales y cuadernos de notas en línea las conoce aproximadamente la mitad de la muestra. Herramientas para la creación colaborativa de documentos, recursos como el Aula Virtual, presentaciones en línea y la blogosfera son concidas por un $85 \%$ aproximadamente. Mientras que las herramientas más conocidas son los programas para la elaboración de presentaciones, procesadores de textos, hojas de cálculo, editores de videos e imágenes, así como las redes sociales que son conocidas por casi toda la población. 


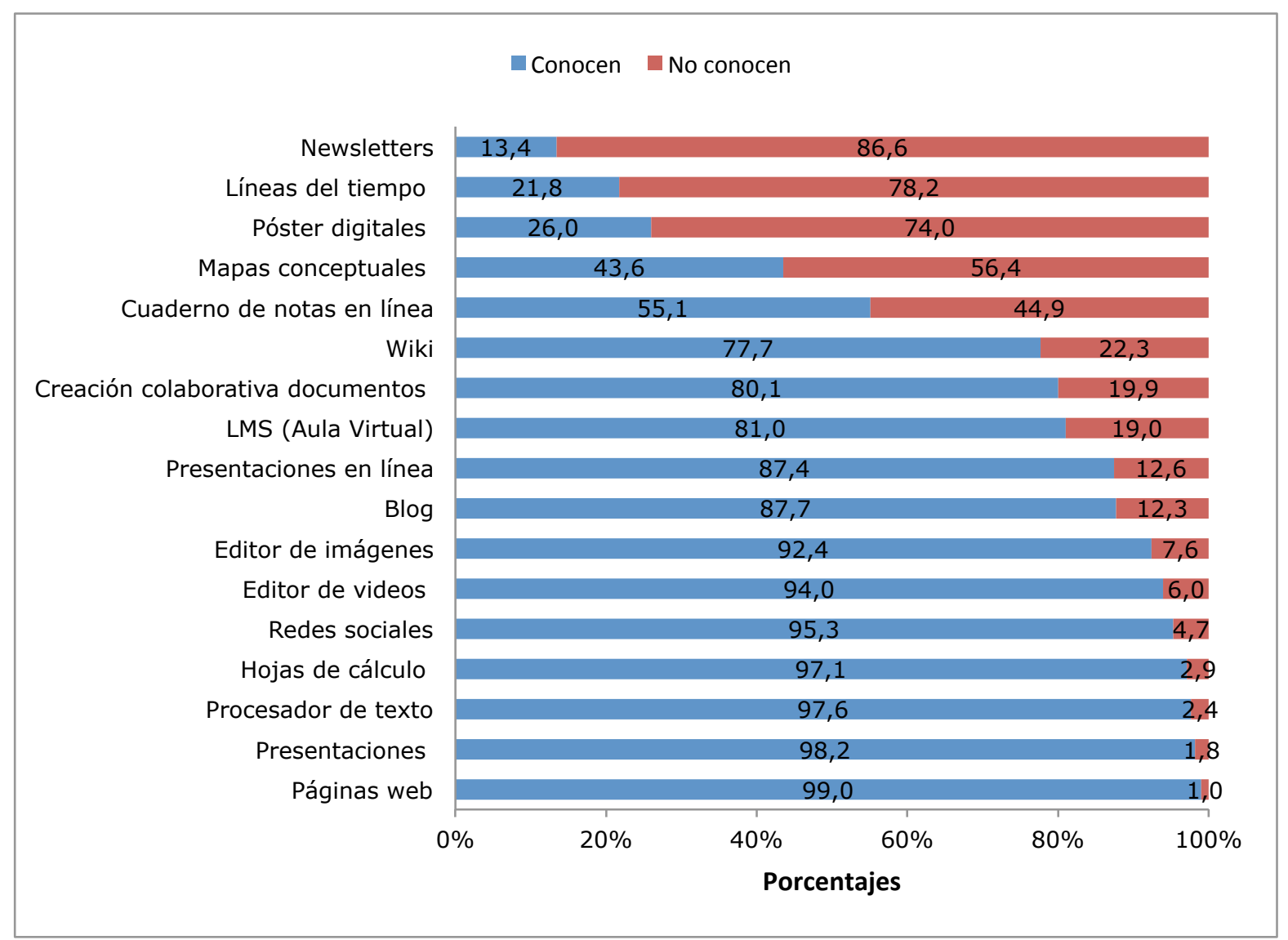

Figura 3. Principales herramientas conocidas por el colectivo discente para la reflexión de la información Fuente: Elaboración propia

\section{b. Principales finalidades que motivan al estudiantado en el uso de herramientas para generar y gestionar el contenido}

Como puede verse en la figura 4, entre las principales finalidades relacionadas con la generación y gestión de contenido, destacan aspectos como organización de la información, creatividad, estructuración de ideas, estudio o aporte teórico, donde entre el $70 \%$ y $80 \%$ del colectivo indica que lo realiza casi siempre o siempre; el trabajo colaborativo un $70 \%$ aproximadamente; y criticidad y síntesis un $60 \%$ de los encuestados. Por último, la mitad de los encuestados indica que siempre o casi siempre emplean las herramientas de generación y gestión de contenidos para suscitar discusiones y debates. 


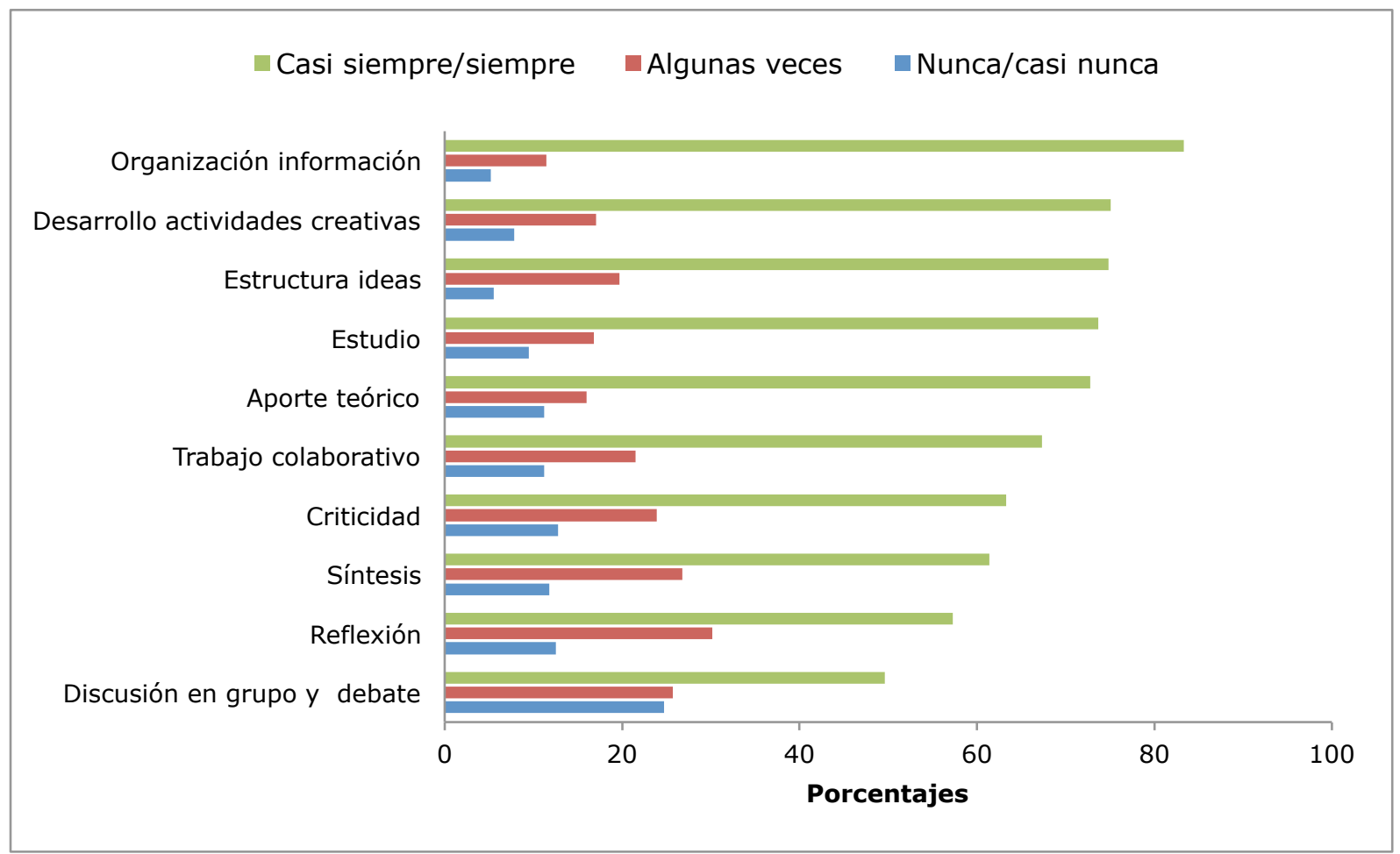

Figura 4. Principales finalidades de la utilización de herramientas para reflexionar

Fuente: Elaboración propia

\section{c. Principales actividades que genera el estudiantado en el uso de herramientas para generar y gestionar contenido}

Entre las actividades que suelen realizar para tratar la información conseguida destacan, según los resultados reflejados en la figura 5, las enfocadas a presentar trabajos para los cursos y elaborar presentaciones: donde el $90 \%$ de las personas encuestadas indican que lo hacen casi siempre o siempre. Por el contrario, para realizar o publicar documentos solamente la mitad dicen hacerlo. Cabe destacar, como ya se ha mencionado, la poca utilización de herramientas en línea para modificar la información o elaborar conocimiento, como se demuestra en actividades como la realización de mapas conceptuales, elaboración de material en línea, realización de videos o diarios de trabajo, donde aproximadamente la mitad de las personas indica que no lo hace nunca o casi nunca. Por último, actividades como creación de líneas del tiempo y posters digitales la gran mayoría (75\%) dice no hacerlo nunca o casi nunca, a pesar de que existen recursos específicos en línea para la realización de estas tareas. 


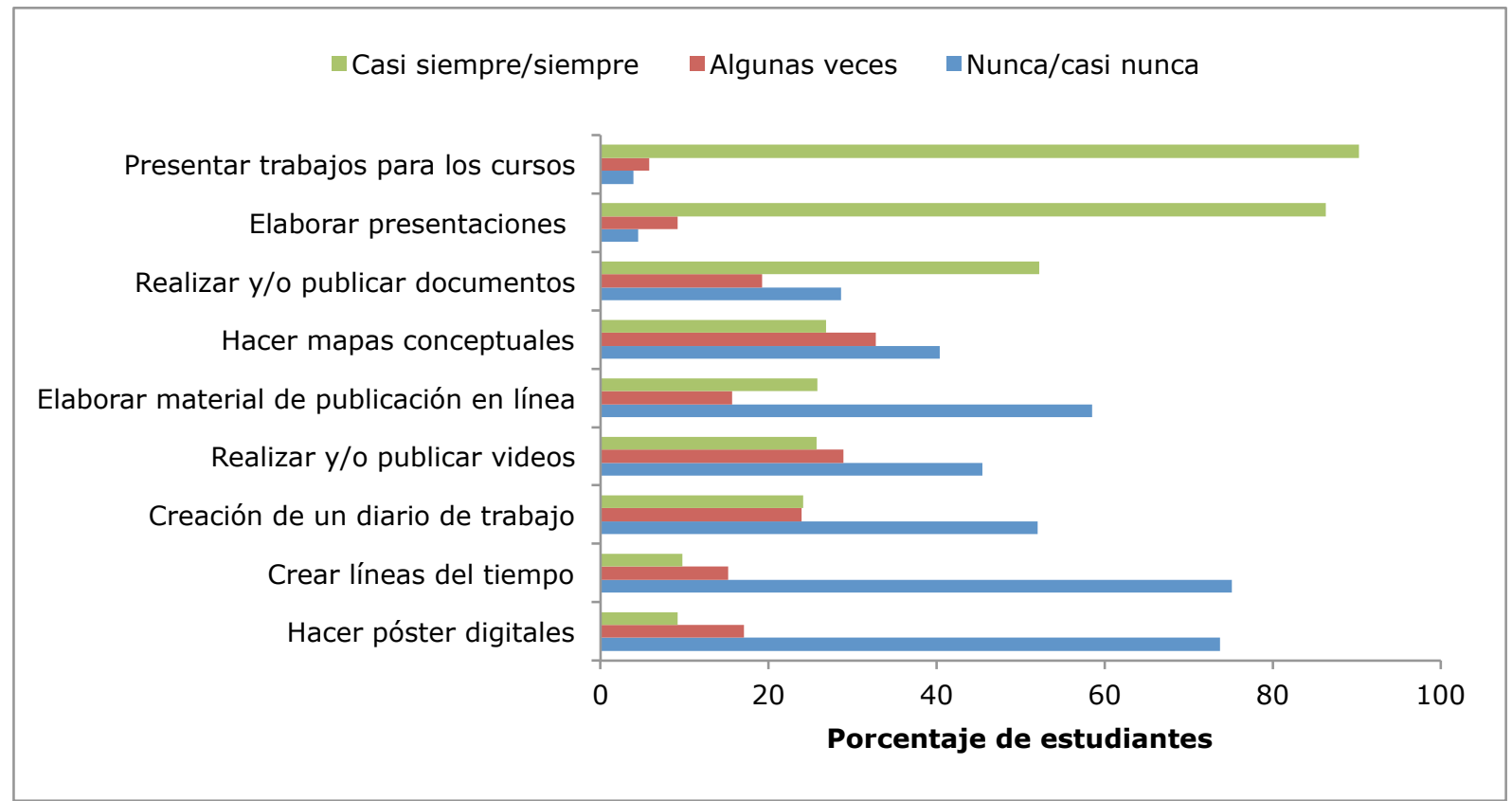

Figura 5. Actividades que genera el estudiantado en la creación de contenidos.

Fuente: Elaboración propia

\section{d. Frecuencia de uso con la que el estudiantado utiliza las herramientas para crear contenido}

Los recursos que el estudiantado utiliza para generar y gestionar el contenido no pertenecen a la ya mencionada web 2.0, como se observa en la tabla 3. Las que han obtenido mayor puntuación con respecto a la media, coincidiendo con las que el colectivo más conoce, son los procesadores de texto (4.58) y herramientas para elaborar presentaciones (4.40). Seguidamente utilizan en mayor medida las redes sociales (3.93), recursos para la elaboración colaborativa de documentos en línea (3.15), programas para la edición de videos (2.98) y hojas de cálculo (2.93). En cuanto a las menos utilizadas cabe destacar Newsletters (1.73), cuaderno de notas en línea (1.82) y blog (2.04). Aunque la página web es muy utilizada para buscar información, el grupo encuestado no la utiliza para generar contenido (2.10). Otras herramientas para la elaboración de póster digitales, líneas del tiempo o wikis obtienen medias poco elevadas, evidenciando la poca utilización que se les otorga para la finalidad que nos ocupa.

\begin{tabular}{|c|c|c|c|c|c|c|c|c|}
\hline \multirow[b]{2}{*}{ Herramientas para reflexionar } & \multicolumn{8}{|c|}{ Frecuencia de uso } \\
\hline & 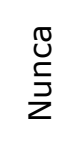 & 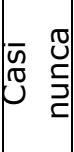 &  &  & $\begin{array}{l}\frac{\varrho}{0} \\
\frac{\varrho}{E} \\
\frac{\omega}{\omega}\end{array}$ & Total & Media & DS \\
\hline Blog & 131 & 92 & 81 & 18 & 9 & 331 & 2.04 & 1.05 \\
\hline Wiki & 116 & 68 & 73 & 36 & 16 & 309 & 2.25 & 1.21 \\
\hline Presentaciones en línea & 86 & 76 & 85 & 51 & 34 & 332 & 2.61 & 1.30 \\
\hline Editor de imágenes & 60 & 72 & 96 & 67 & 56 & 351 & 2.96 & 1.31 \\
\hline Páginas web & 132 & 84 & 69 & 42 & 48 & 375 & 2.10 & 1.39 \\
\hline Cuaderno de notas en línea & 112 & 45 & 37 & 8 & 7 & 209 & 1.82 & 1.07 \\
\hline
\end{tabular}




\begin{tabular}{|l|c|c|c|c|c|c|c|c|}
\hline Newsletters & 32 & 10 & 3 & 2 & 3 & 50 & 1.73 & 1.16 \\
\hline Creación colaborativa documentos & 56 & 49 & 64 & 62 & 73 & 304 & 3.15 & 1.43 \\
\hline Póster digitales & 41 & 15 & 20 & 6 & 14 & 96 & 2.47 & 1.93 \\
\hline Procesador de texto & 13 & 10 & 18 & 38 & 292 & 371 & 4.58 & 0.96 \\
\hline Hojas de cálculo & 66 & 83 & 100 & 49 & 71 & 369 & 2.93 & 1.36 \\
\hline Editor de videos & 53 & 84 & 103 & 51 & 66 & 357 & 2.98 & 1.31 \\
\hline Presentaciones & 10 & 9 & 30 & 96 & 228 & 373 & 4.40 & 0.93 \\
\hline Líneas del tiempo & 36 & 17 & 14 & 8 & 7 & 82 & 2.18 & 1.32 \\
\hline Mapas conceptuales & 41 & 37 & 54 & 20 & 13 & 165 & 2.56 & 1.21 \\
\hline LMS (Aula Virtual) & 48 & 66 & 89 & 45 & 45 & 293 & 2.90 & 1.28 \\
\hline Redes sociales & 36 & 26 & 57 & 67 & 191 & 377 & 3.93 & 1.34 \\
\hline
\end{tabular}

Tabla 3. Frecuencias de uso en herramientas para crear contenido

Fuente: Elaboración propia

\section{e. Percepción sobre el nivel de capacitación de los estudiantes en el uso de herramientas tecnológicas para generar y gestionar el contenido}

De las herramientas que el estudiantado utiliza para crear contenido, cabe mencionar el bajo nivel de capacitación. Como se observa en la figura 6, solamente destaca el dominio en procesadores de texto y elaboración de presentaciones ( $82 \%)$, así como en las redes sociales (73\%) y páginas web $(64 \%)$. Consideran tener una capacitación media en hojas de cálculo y presentaciones en línea (55\% aproximadamente), a pesar de ser herramientas bastante utilizadas (ver tabla 2). En cuanto a los recursos en los que el grupo discente reconoce estar menos capacitado, cabe mencionar el blog y el editor de imágenes (55\%), cuaderno de notas en línea y newsletters (52\%) y el Aula Virtual (LMS) donde casi la mitad de las personas indica esta opción. Destacar que en cuanto a las herramientas en línea para la elaboración de posters digitales, líneas del tiempo y mapas conceptuales, el colectivo indica estar muy capacitado en torno a un $45 \%$, mientras manifiestan estar poco capacitados cerca del 50\%, reduciéndose la capacitación media a menos del $10 \%$. 


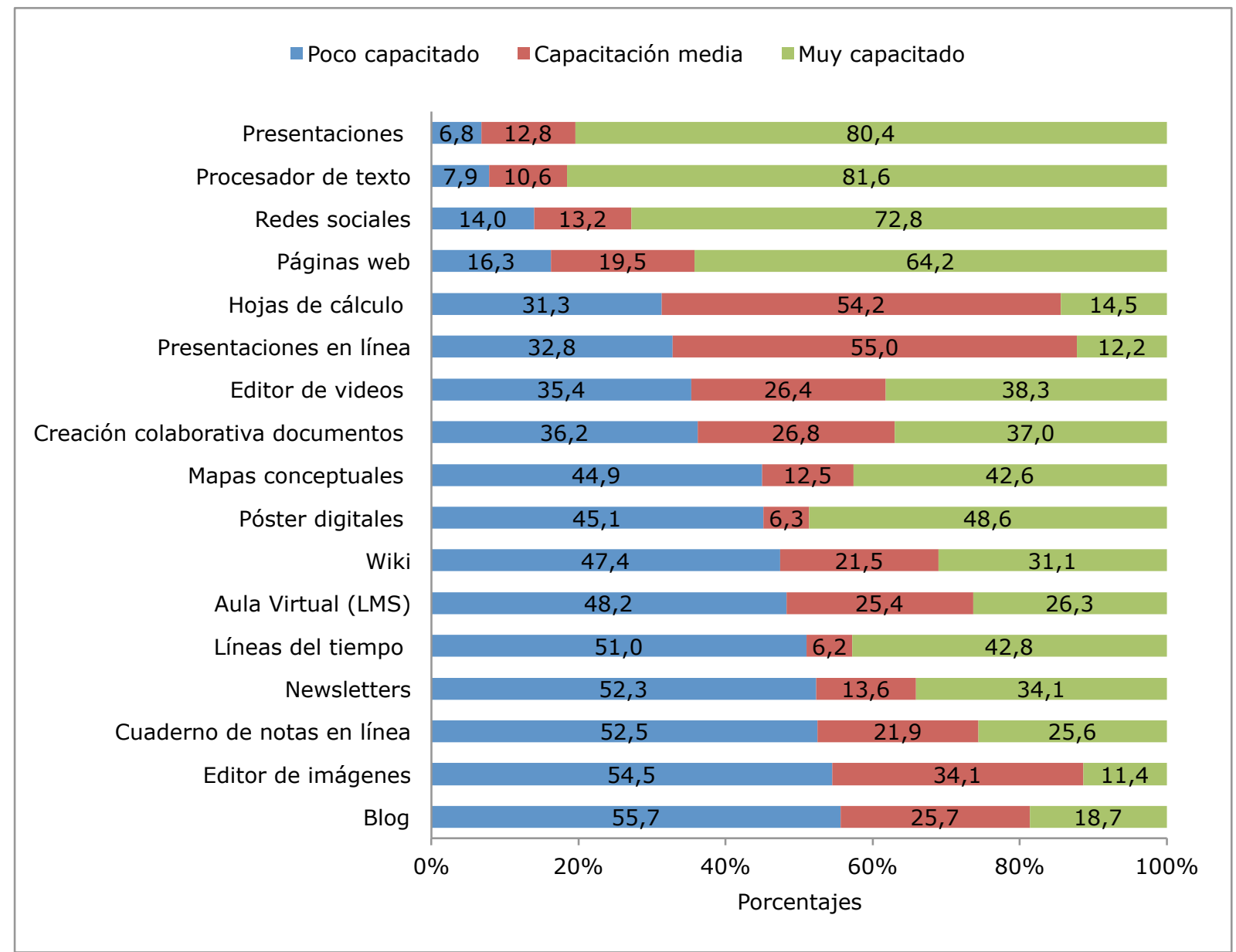

Figura 6. Percepción del estudiantado sobre capacitación en herramientas para buscar información Fuente: Elaboración propia

\section{f. Análisis de diferencias en función de la variable sexo}

La comprobación de la normalidad se realizó a través del contraste Kolmogorov-Smirnov, obteniendo un nivel " $p$ " no significativo ( $p>.05$ ) para la variable sexo (hombres y mujeres).

Igualmente se verificó la homogeneidad de varianzas, empleando para ello la prueba de Levene ( $\mathrm{p}$ valor $=0.433$ ). Cumplidos, por tanto, los requisitos de normalidad y homocedasticidad, en la prueba $t$ propiamente dicha de igualdad de medias (tabla 4), no se han encontrado diferencias significativas en las puntuaciones entre los hombres $(M=128,16 ; S D=31.77)$ y mujeres $[M=$ $122,76 ; \mathrm{SD}=29.72 ; \mathrm{t}(363)=1,23, \mathrm{p}=0,217]$. Por lo que se puede afirmar que no existen diferencias en cuanto a la variable "generar y gestionar contenido" en función del sexo. 
Prueba T para la igualdad de medias

\begin{tabular}{|c|c|c|c|c|c|c|c|}
\hline & \multirow{2}{*}{\multicolumn{2}{|c|}{$\mathrm{gl}$}} & \multirow[t]{2}{*}{$\begin{array}{c}\text { Sig. } \\
\text { (bilateral) }\end{array}$} & \multirow[t]{2}{*}{$\begin{array}{c}\text { Diferencia } \\
\text { de } \\
\text { medias }\end{array}$} & \multirow[t]{2}{*}{$\begin{array}{l}\text { Error típ. } \\
\text { de la } \\
\text { diferencia }\end{array}$} & \multicolumn{2}{|c|}{$\begin{array}{c}\text { 95\% Intervalo de } \\
\text { confianza para la } \\
\text { diferencia }\end{array}$} \\
\hline & & & & & & Inferior & Superior \\
\hline $\begin{array}{l}\text { Escala generar y } \\
\text { gestionar el contenido }\end{array}$ & 1,237 & 363 & 217 & 5,39696 & 4,36317 & $-3,183$ & 13,977 \\
\hline
\end{tabular}

Tabla 4. Prueba t de Student para la igualdad de medias. Factor de agrupación: "sexo".

Fuente: Elaboración propia

\section{g. Análisis de diferencias en función de la variable edad}

Teniendo en cuenta los resultados de las pruebas de normalidad Kolmogorov-Smirnov y ShapiroWilk ( $p>05)$, así como la homogeneidad de varianzas con la prueba de Levene con una significación de 0.673 , se opta por realizar la prueba paramétrica ANOVA de un factor con pruebas post-hoc, donde no se han encontrado diferencias significativas, según se observa en la tabla 5.

\begin{tabular}{llllllr}
\hline & & $\begin{array}{c}\text { Suma de } \\
\text { cuadrados }\end{array}$ & gl & $\begin{array}{c}\text { Media } \\
\text { cuadrática }\end{array}$ & $\mathrm{F}$ & Sig. \\
\hline $\begin{array}{l}\text { Generar y gestionar el } \\
\text { contenido }\end{array}$ & $\begin{array}{l}\text { Inter- } \\
\text { grupos }\end{array}$ & 3112,074 & 3 & 1037,358 & 1,149 &, 329 \\
\cline { 2 - 7 } & $\begin{array}{l}\text { Intra- } \\
\text { grupos }\end{array}$ & 325884,101 & 361 & 902,726 & & \\
\cline { 2 - 7 } & Total & 328996,175 & 364 & & \\
\hline
\end{tabular}

Tabla 5. ANOVA. Factor de agrupación: "edad".

Fuente: Elaboración propia

\section{h. Análisis de diferencias en función de la variable acceso a recursos tecnológicos}

Después de comprobar la normalidad y homogeneidad, se han realizado pruebas $t$ de Student para muestras independientes (ver tabla 6), concretamente tomando en cuenta la posesión de computadora propia y conexión a internet en el lugar de residencia durante los estudios. En el primero de los casos no se han hallado diferencias entre los que indican tener computadora propia $(M=123,69 ; S D=30,40)$ y los que no $[M=124,00 ; S D=22,26 ; t(362)=0.36, p=0,971]$. Por el contrario, en el segundo supuesto sí se han encontrado diferencias entre los que indican tener acceso a internet $(M=125,25 ; S D=29,72)$ y los que no $[M=102,89 ; S D=26,95 ; t$ t $(363)=$ $3,79, p=0,000]$, pudiendo afirmar en base a este estadístico que existen diferencias significativas en la variable referida al acceso a la conexión a internet. 


\begin{tabular}{|c|c|c|c|c|c|c|c|}
\hline & \multicolumn{7}{|c|}{ Prueba T para la igualdad de medias } \\
\hline & \multirow[t]{2}{*}{$\mathrm{t}$} & \multirow[t]{2}{*}{$\mathrm{gl}$} & \multirow[t]{2}{*}{$\begin{array}{c}\text { Sig. } \\
\text { (bilateral) }\end{array}$} & \multirow[t]{2}{*}{$\begin{array}{c}\text { Diferencia } \\
\text { de } \\
\text { medias }\end{array}$} & \multirow[t]{2}{*}{$\begin{array}{l}\text { Error típ. } \\
\text { de la } \\
\text { diferencia }\end{array}$} & \multicolumn{2}{|c|}{$\begin{array}{c}\text { 95\% Intervalo de } \\
\text { confianza para la } \\
\text { diferencia }\end{array}$} \\
\hline & & & & & & Inferior & Superior \\
\hline $\begin{array}{l}\text { Posesión de } \\
\text { computadora propia }\end{array}$ &,- 036 & 362 & 971 &,- 30484 & 8,49215 & $-17,0049$ & 16,39531 \\
\hline $\begin{array}{l}\text { Conexión a internet } \\
\text { en el lugar de } \\
\text { residencia }\end{array}$ & 3,786 & 363 & ,000 & 22,35667 & 5,90526 & 10,74385 & 33,96950 \\
\hline
\end{tabular}

Tabla 6. Prueba t de Student para la igualdad de medias. Factor de agrupación: "acceso a recursos tecnológicos".

Fuente: Elaboración propia

\section{i. Análisis de diferencias en función de la variable formación en TIC}

En lo que respecta a la variable formación en TIC, se presentan en la tabla 7, los estadísticos de grupo y los resultados de las pruebas $t$ de Student, donde se pueden observar diferencias significativas entre los que indican haber recibido un curso sobre TIC fuera de Secundaria o de la Universidad (profesor particular, academia, etc.) y los que afirman no haberlo recibido. Igualmente ocurre con aquellas personas que dicen haber recibido formación en Secundaria con respecto a los que niegan haberlo hecho. En el caso de formación sobre TIC en la Universidad, no se han encontrado diferencias significativas, aunque cabe resaltar que el estudiantado que sí ha sido formado obtiene una media considerablemente superior con respecto a los que no han obtenido formación.

En lo que respecta a la percepción que el estudiantado tiene sobre el uso de las TIC para su autoformación, se encuentran diferencias significativas, obteniendo una media muy superior los que indican sí utilizarlo para este fin respecto a los que niegan hacerlo.

\begin{tabular}{|c|c|c|c|c|c|c|}
\hline \multirow{2}{*}{$\begin{array}{l}\text { Variable: "formación } \\
\text { en TIC" }\end{array}$} & \multicolumn{3}{|c|}{ Estadísticos de grupo } & \multicolumn{3}{|c|}{$\begin{array}{c}\text { Prueba T para la igualdad } \\
\text { de medias }\end{array}$} \\
\hline & Respuesta & Media & $\begin{array}{l}\text { Desviación } \\
\text { típica }\end{array}$ & $\mathrm{t}$ & gl & $\begin{array}{l}\text { Sig. } \\
\text { (bilateral) }\end{array}$ \\
\hline \multirow{2}{*}{\begin{tabular}{|l|} 
Curso TIC \\
independiente
\end{tabular}} & Sí & 129,90 & 32,73 & \multirow{2}{*}{2,923} & \multirow{2}{*}{362} & \multirow{2}{*}{,004 } \\
\hline & No & 120,30 & 28,11 & & & \\
\hline \multirow{2}{*}{$\begin{array}{l}\text { Curso TIC } \\
\text { Secundaria }\end{array}$} & Sí & 127,93 & 28,60 & \multirow{2}{*}{2,441} & \multirow{2}{*}{361} & \multirow{2}{*}{,015 } \\
\hline & No & 120,31 & 30,53 & & & \\
\hline \multirow{2}{*}{$\begin{array}{l}\text { Curso TIC } \\
\text { Universidad }\end{array}$} & Sí & 125,95 & 30,75 & \multirow{2}{*}{1,882} & \multirow{2}{*}{360} & \multirow{2}{*}{,061 } \\
\hline & No & 119,84 & 27,80 & & & \\
\hline \multirow{2}{*}{$\begin{array}{l}\text { Percepción auto } \\
\text { formación }\end{array}$} & Sí & 124,44 & 29,92 & \multirow{2}{*}{3,067} & \multirow{2}{*}{361} & \multirow{2}{*}{,002 } \\
\hline & No & 96,45 & 25,16 & & & \\
\hline
\end{tabular}

Tabla 7. Estadísticos de grupo y resultados de las pruebas t de Student de formación en TIC y escala PLE

Fuente: Elaboración propia 


\section{Discusión y conclusiones}

No cabe duda que la alfabetización digital incorpora la producción de contenido como una de sus premisas fundamentales (Erstad, 2010). Además, al formar parte de la estructura del PLE (Adell y Castañeda, 2010), la forma en que se genera y gestiona la información, hace que los entornos del estudiantado se vean potencializados a través de las múltiples y diferentes posibilidades que ofrecen los recursos tecnológicos, especialmente de la web 2.0. Analizar los PLE de futuros formadores en su etapa formativa de último año, ayudará a entender su proceso de aprendizaje, así como las herramientas utilizadas, las finalidades perseguidas y las actividades generadas, nutridas tanto en su paso por la universidad así a través de otros espacios de formación. $Y$, al mismo tiempo, permite conocer si la universidad desarrolla competencias digitales en el colectivo discente durante su proceso formativo (Gisbert y Esteve, 2016)

De los datos obtenidos en el proceso de investigación, se han identificado las principales herramientas que el estudiantado de último año de carrera del CIDE conoce y utiliza para crear y gestionar el contenido, siendo las aplicaciones de escritorio para el desarrollo de presentaciones, procesadores de texto, hojas de cálculo y editor de videos, las más comunes. Queda de manifiesto el poco conocimiento, y por ende la poca utilización, que se posee sobre ciertas herramientas de la web 2.0 para la elaboración de líneas temporales, pósters digitales, mapas conceptuales, o la reflexión en blogs y wikis, no aprovechando las ventajas de estas herramientas de carácter gratuito y accesibles. Esta circunstancia cuestiona la alfabetización del estudiantado en cuanto a la generación de contenido, concordando con lo ya manifestado por Erstad (2010). Pero además, pone en duda la posibilidad de que el estudiante, futuro maestro, pueda convertirse en agente de cambio, y que se anime y se comprometa con el propósito y la tarea de apoyar y facilitar las competencias tecnológicas necesarias a los que serán sus estudiantes una vez acceda a la docencia (Roig et al., 2015; Almenara, et al, 2017).

Algunos trabajos (Atwell, 2007; Cabero y Marín, 2011; Ruiz et al., 2013; Venkatesh et al., 2014, Boza y Conde, 2015) identifican ciertas herramientas para la creación de contenidos que fomentan el desarrollo de los PLE, entre los que destacan blogs y wikis, recursos que la gran mayoría del estudiantado del CIDE conoce pero no utiliza, coincidiendo así con otros estudios (Sahin y Uluyol, 2016; Vicent et al., 2017), donde los principales recursos que utilizan son para la búsqueda e intercambio de información, y no para la creación de contenidos.

Si bien es cierto, que la mayoría del estudiantado accede a la educación superior con cierta alfabetización digital (Gisbert y Esteve, 2016), estudios como el de García et al. (2016), muestran los escasos recursos que utilizan antes de su ingreso a la universidad, siendo en su mayoría redes sociales que no suelen asociarse a funciones educativas sino de ocio. De lo anterior se desprende que la Universidad no está desarrollando los PLE del estudiantado en cuanto a herramientas tecnológicas, aspecto que dificulta la descentralización de estrategias didácticas desde la institución al estudiante, para que pueda orientar su propio aprendizaje de acuerdo a sus necesidades, y puedan articularse alternativas viables disruptivas frente a la estructura jerárquica del entorno formativo tradicional (Fernández y Cejudo, 2009).

Teniendo en cuenta que no solamente las herramientas utilizadas componen los PLE (Adell y Castañeda, 2010), a través de este estudio, también se han identificado las principales finalidades y actividades que el estudiantado incorpora en sus PLE. En cuanto a las finalidades se observa como incorporan en mayor medida la organización de información, aspectos de creatividad, 
estructuración de ideas o trabajo colaborativo, aprovechando ciertas potencialidades de algunas herramientas. Por otro lado, se observa un detrimento en los PLE respecto a la realización de actividades que en su gran mayoría se centran en aspectos relacionados con las exigencias de un currículo más tradicional y transmisivo, y que se resumen en la elaboración de documentos en procesadores de texto y presentaciones. Ante este panorama surge la pregunta crealmente se están generando cambios y replanteamientos sobre la forma de enseñar y aprender?

El nivel de capacitación que tiene el estudiantado en el manejo de herramientas para crear contenido puede considerarse bajo, coincidiendo con estudios realizados con muestras de similares características, como el de Colmenero et al. (2015). Concretamente, se constata que solamente tienen una buena capacitación en algunas de las herramientas como procesadores de texto, para elaborar presentaciones y en el manejo de redes sociales. Si bien algunas de las carreras ofrecen cursos específicos sobre tecnología educativa en los primeros años, esta falta de capacitación puede interpretarse desde dos vertientes, por un lado y a la luz de los resultados, se observa la ausencia de iniciativa del estudiantado para la incorporación dentro de sus PLE de herramientas de la web 2.0, los cual hace que ante el bajo nivel de capacitación, la frecuencia de uso sea escasa. Por otro lado, también hay que apelar a las exigencias de los cursos a lo largo de la formación universitaria y/o a los requerimientos y motivaciones desarrolladas por el colectivo docente, que de una u otra manera, no aportan herramientas y actividades nuevas a los PLE del estudiantado, afectando de manera significativa al nivel de capacitación.

No se han encontrado diferencias significativas en las puntuaciones obtenidas de la subescala creación y gestión del contenido respecto a la variable género, aunque cabe resaltar, que en el caso de las mujeres las puntuaciones son menores que en el grupo de los hombres. Estos hallazgos contradicen a los obtenidos en otros estudios (Venkatesh et al. 2014), que sí encontraron diferencias en el uso de tecnologías, quizás porque ciertos estereotipos de género y algunos aspectos culturales podrían ser factores incidentes. Igualmente, no se han encontrado diferencias significativas en la variable edad, coincidiendo con Escofet et al. (2014) al indicar que nacer en la era de las nuevas tecnologías no deriva en habilidad para el uso académico de las mismas.

En cuanto al acceso a recursos tecnológicos, es importante resaltar que la gran mayoría (96.3\%) posee computadora propia, así como acceso a internet en su lugar de residencia (92.9\%). Los resultados arrojan que no existen diferencias significativas entre el grupo que indica tener equipo propio y el que no. Por el contrario, sí se han encontrado diferencias significativas según el acceso a internet, en el sentido de que aquellos que tienen acceso a internet obtienen mayor puntuación en el componente creación de contenido, lo que deriva en un PLE más desarrollado.

Por último, la variable formación en TIC presenta diferencias significativas, en el sentido de que aquellas personas que han sido formadas previamente a su ingreso a la universidad, tanto con cursos en la secundaria o fuera de la educación formal, tienen un PLE más desarrollado que los que no han recibido dicha formación. Estos resultados muestran la importancia de la formación en TIC, particularmente a través de cursos específicos, ya que como indica Comenero et al. (2015) la transversalización tecnológica no siempre es real.

De manera general este artículo ofrece una perspectiva sobre las herramientas que el estudiantado de últimos años de carreras de Educación utiliza para generar nuevo contenido o modificar información existente. Queda en evidencia el escaso conocimiento y uso que hacen de recursos que 
facilitan la creación de contenido en diferentes formatos como el multimedia, prefiriendo las herramientas de escritorio, y como estas limitaciones pueden derivar en un PLE poco desarrollado y, por ende, en un aprendizaje todavía centralizado en la institución formadora y alcance restringido.

\section{Agradecimientos}

Este artículo se ha elaborado en el marco del proyecto de investigación titulado: "Ecologías de aprendizaje en la era digital: nuevas oportunidades para la formación del profesorado de educación secundaria" (ECO4LEARN-SE), parcialmente financiado por el Ministerio de Ciencia, Innovación y Universidades (Referencia RTI2018-095690-B-I00)

\section{Referencias}

Adell, J. y Castañeda, L. (2010). "Los Entornos Personales de Aprendizaje (PLES): una nueva manera de entender el aprendizaje". En Roig Vila, R. y Fiorucci, M. (Eds.) Claves para la investigación en innovación y calidad educativas. La integración de las Tecnologías de la Información y la Comunicación y la Interculturalidad en las aulas. Stumenti di ricerca per l'innovaziones e la qualità in ámbito educativo. La Tecnologie dell'informazione e della Comunicaziones e l'interculturalità nella scuola. Alcoy: Marfil - Roma TRE Universita degli studi.

Ahmed, Abdelrahman M., AbdelAlmuniem, Arwa, \& Almabhouh, Ahmed A. (2016). The Current Use of Web 2.0 Tools in University Teaching from the Perspective of Faculty Members at the College of Education. International Journal of Instruction, 9(1), 179-194.

Arnal, J., Del Rincón, D., \& Latorre, A. (1992). Investigación educativa. Fundamentos y metodología. Barcelona: Labor.

Arul Sekar, J.M., \& Arul Lawrence, A.S. (2015). Attitude of B.Ed. students towards information and communication technology (ICT). International Journal of Applied Research; 1(8): 785-787. Recuperado de http://www.allresearchjournal.com/archives/2015/vol1issue8/PartM/1-8-167.pdf

Attwell, G. (2007). Personal learning environments-the future of elearning. Elearning Papers, 2 (1), 1-7.

Barboza, E. C., Torres, J. M. T., \& Núñez, J. A. L. (2016). Acciones para la autorregulación del aprendizaje en entornos personales. Pixel-Bit: Revista de medios y educación, (48), 67-82.

Bisquerra, R. (Coord.). (2014). Metodología de la investigación educativa. Madrid, España: Editorial La Muralla.

Boza, A. \& Conde, S. (2015). Web 2.0 en educación superior: formación, actitud, uso, impacto, dificultades y herramientas. In: Digital Education Review, 28, 45-58. Recuperado de http://greav.ub.edu/der

Buendía, L.,Olmedo, E.M y González, G. (2009). Lifelong learning: diferentes contextos, diferentes situaciones. Revista de Investigación Educativa, 27 (1), 185-202.

Cabero, J. y Marín, V. (2011).Creación de un entorno personal para el aprendizaje: desarrollo de una experiencia. Edutec-e, Revista Electrónica de Tecnología Educativa, 38. Recuperado de http://edutec.rediris.es/Revelec2/Revelec38/creacion_entorno_personal_aprendizaje_desarrollo_experi encia.html

Almenara, J. C., Roig-Vila, R., \& Mengual-Andrés, S. (2017). Conocimientos tecnológicos, pedagógicos y disciplinares de los futuros docentes según el modelo TPACK. Digital Education Review, (32), 73-84. Recuperado de https://goo.gl/gT5F4Z

Castañeda, L. y Adell, J. (2011). El desarrollo profesional de los docentes en entornos personales de aprendizaje (PLE). En Roig Vila, R. y Laneve, C. (Eds.) La práctica educativa en la Sociedad de la Información: Innovación a través de la investigación / La pratica educativa nella Società dell'informazione: L'innovazione attraverso la ricerca. Alcoy: Marfil. 83-95

Castañeda, L. y Adell, J. (Eds.). (2013). Entornos Personales de Aprendizaje: claves para el ecosistema educativo en red. Alcoy: Marfil. 
Cómo generan y gestionan contenidos los estudiantes de educación de Costa Rica: una contribución al estudio de su entorno personal de aprendizaje

Cohen, L., \& Manion, L. (1990). Métodos de investigación educativa. Madrid: La Muralla.

Colmenero, M. J. R., Pérez, M. C. S., \& Gutiérrez, R. C. (2015). El reto de la competencia digital en los futuros docentes de Infantil, Primaria y Secundaria: los estudiantes de grado y máster de Educación ante las TIC. Prisma Social: revista de ciencias sociales, (15), 254-295.

Cronbach, L.J. (1951). Coefficiente alfa and the internal structures of tests [Coeficiente Alfa y estructura interna de los test]. Psychometrika, 16 (3), 297-334. Retrieved from http://psych.colorado.edu/ carey/courses/psyc5112/readings/alpha_cronbach.pdf

Dabbagh, N., \& Reo, R. (2011). Impact of Web 2.0 on higher education. In D. W. Surry, T. Stefurak, \& R. Gray (Eds.), Technology integration in higher education: Social and organizational aspects (pp.174-187). Hershey, PA: IGI Global. doi: 10.4018/978-1-60960-147-8.ch013

Erstad, O. (2015). Educating the Digital Generation - Exploring Media Literacy for the 21st Century Nordic Journal of Digital Literacy, (4), 85-102. Retrieved from https://www.researchgate.net/publication/284918764

Escofet Roig, A., López, M., y Álvarez, G. (2014). Una mirada crítica sobre los nativos digitales: análisis de los usos formales de tic entre estudiantes universitarios. Revista Q, Vol. 9 Issue 17, p1-18. Recuperado de https://goo.gl/cFKyYv

Fernández, G. D., \& Cejudo, M. D. C. L. (2009). La Educación Social y la web 2.0: nuevos espacios de innovación e interacción social en el Espacio Europeo de Educación Superior. Pixel-Bit: Revista de medios y educación, (35), 105-114. Recuperado de https://dialnet.unirioja.es/servlet/articulo?codigo $=3040873$

Fiedler, S. \& Väljataga, T. (2010). Personal learning environments: Concept or technology?[Entorno Personal de Aprendizaje: ¿concepto o tecnología?. PLE Conference 2010. Retrieved from: http://pleconference.citilab.eu

Fiedler, S. H. D., \& Väljataga, T. (2013). Personal learning environments: A conceptual landscape revisited. eLearning Papers, 35, 1-16.

Gallego-Arrufat, M.J. y Chaves-Barboza, E. (2014). Tendencias en estudios sobre entornos personales de aprendizaje (Personal Learning Environments -PLE-). EDUTEC, Revista Electrónica de Tecnología Educativa, 49. Recuperado de http://edutec.rediris.es/Revelec2/Revelec49/n49_Gallego_Chaves.html

Gisbert, M., \& Esteve, F. (2016). Digital Leaners: la competencia digital de los estudiantes universitarios. La cuestión universitaria, (7), 48-59. Recuperado de http://polired.upm.es/index.php/lacuestionuniversitaria/article/view/3359

Harris, L., Earl, G., Beale, N., Phethean, C., \& Brughmans, T. (2012). Building personal learning networks through event-based social media: A case study of the SMiLE project. In PLE Conference Proceedings (Vol. 1, No. 1).

Hernández, R; Fernández, C y Baptista, P. (2010). Metodología de la investigación. México D.F., México: Editorial Mc Graw Hill.

Johnson, M., \& Liber, O. (2008). The Personal Learning Environment and the human condition: From theory to teaching practice. Interactive Learning Environments, 16(1), 3-15. doi:10.1080/10494820701772652

Kop, R., \& Fournier, H. (2013). Developing a framework for research on personal learning environments. eLearning Papers, www.openeducationeuropa. eu/en/elearning_papers

Marín V.I., Lizana, A. y Salinas, J. (2014). Cultivando el PLE: una estrategia para la integración de aprendizajes en la universidad. EDUTEC, Revista Electrónica de Tecnología Educativa, 47. Recuperado de http://edutec.rediris.es/Revelec2/Revelec47/n47_Marin-Lizana-Salinas.html

García, J. A., Fallas, M. A., \& Gamboa, A. (2016). Desarrollo del entorno personal de aprendizaje: valoración de una experiencia con estudiantes universitarios. Summa Psicológica UST, 13(2), 83-94. DOI: http://dx.doi.org/10.18774/summa-vol13.num2-317

Rahimi, E., Berg, J. \& Veen, W. (2012). Designing and Implementing PLEs in a Secondary School Using Web2.0 Tools [Diseño e implementación de PLEs in una Escuela de Secundaria usando herramientas de la web 2.0]. PLE Conference 2012. Recuperado de http://revistas.ua.pt/index.php/ple/article/view/1456 
Rahimi, E., Berg, J., \& Veen, W. (2015). Facilitating student-driven constructing of learning environments using Web 2.0 personal learning environments. Computers \& Education 81(2015),235-246. Recuperado de http://www.sciencedirect.com/science/article/pii/S0360131514002322

Roig Vila, R., Mengual-Andrés, S., Sterrantino, C. \& Quinto, P. (2015). Actitudes hacia los recursos tecnológicos en el aula de los futuros docentes. @tic. Revista D'innovació Educativa, (15), 12-19.

Ruiz, J., Sánchez, J. y Gómez-García, M. (2013). Entornos Personales de Aprendizaje: Situación en la Facultad de Ciencias de la Educación de la Universidad de Málaga. Pixel Bit. Revista de Medios Y Educación, 42, 171-181. Recuperado de http://acdc.sav.us.es/pixelbit/images/stories/p42/13.pdf

Saadatmand, M., \& Kumpulainen, K. (2012). Content aggregation and knowledge sharing in a personal learning environment: Serendipitous and emergent learning in open online networks. In2012 15th International Conference on Interactive Collaborative Learning (ICL) (pp. 1-8). doi:10.1109/ICL.2012.6402224

Şahin, S., \& Uluyol, Ç. (2016). Preservice Teachers' Perception and Use of Personal Learning Environments (PLEs). The International Review of Research in Open and Distributed Learning, 17(2). Recuperado de http://www.irrodl.org/index.php/irrodl/article/view/2284

Torres, R. y Costa, C. (2013). Formación continua, aprendiza a lo largo de la vida y PLEs. En Castañeda, L. y Adell, J. (Eds.). (2013). Entornos Personales de Aprendizaje: claves para el ecosistema educativo en red. Alcoy: Marfil.

Väljataga, T., y Laampere, M. (2010). Learner control and personal learning environment: a challenge for instructional design. Interactive Learning Environments, 18(3), 277-292.

Van Den Beemt, A., \& Diepstraten, I. (2016). Teacher perspectives on ICT: A learning ecology approach. Computers and Education, 92-93, 161-170.

Vazquez-Cano E., Martín-Monje, E. \& Castrillo de Larreta-Azelain, M.D. (2016). Analysis of PLEs' Implementation under OER design as a productive teaching-learning strategy in Higher Education. A case study at Universidad Nacional de educación a Distancia. In: Digital Education Review, 29, 62-85. [Accessed: 06/05/2018] http://greav.ub.edu/der

Venkatesh, V., Croteau, A.M., \& Rabah, J. (2014). Perceptions of Effectiveness of Instructional Uses of Technology in Higher Education in an Era of Web 2.0. 47th Hawaii International Conference on System Science. Recuperado de http://ieeexplore.iee.. rg/stamp/stamp.jsp?tp=\&arnumber $=6758617$

Vicent, P. L., Calatayud, V. G., Perera, M. V. A., \& Rodríguez, J. A. (2017). La gestión de la información en entornos personales de aprendizaje: estudio exploratorio en alumnado de último curso de grado. Revista Complutense de Educación, 28(4), 133. Recuperado de https://goo.gl/Lojb4c

Voogt, J. (2010). Teacher factors associated with innovative curriculum goals and pedagogical practices: Differences between extensive and non-extensive ICT-using science teachers. Journal Of Computer Assisted Learning, 26(6), 453-464. 